\title{
Fatigue State Detection From Multi-features
}

\author{
Yuan Gao \\ School of Computer Science and Engineering \\ Xi'an Technological University \\ Xi'an, 710032, China \\ e-mail: 15686060259@163.com
}

\author{
Changyuan Wang \\ School of Computer Science and Engineering \\ Xi'an Technological University \\ Xi'an, 710032, China \\ e-mail: 13991238983@163.com
}

\begin{abstract}
With the quickening pace of modern life and the increasing of work pressure, accidents caused by fatigue problems occur more and more frequently. Developing a highperformance fatigue monitoring technology can not only improve the driver's work efficiency, but also solve the security risks caused by fatigue driving. This paper presents an algorithm of fatigue state detection from multi-features, which can determine whether a driver is in a state of fatigue. The thesis focuses on a non-contact, real-time fatigue detection method based on video, and proposes an algorithm with multiple fatigue characteristics. Firstly, it collects the video through the camera and carries out simple preprocessing. Then, the face area is quickly located by AdaBoost and the face shape model is constructed by ASM, which is used for locating the eye and mouth precisely, and extracting the relevant parameters. Based on the above indicators, it establishes the mapping relation between the characteristic space and fatigue space to judge the status with the SVM. Experiment results show the efficiency of the proposed method.
\end{abstract}

Keywords-Fatigue Detection; Face Detection; Active Shape Model; Support Vector Machine

\section{INTRODUCTION}

In recent years, the problem of fatigue driving has attracted more and more attention. Methods of fatigue detection are no longer just by the way of questionnaires. There are two assessment methods at present for monitoring fatigue[1]: subjective method and objective method. Subjective assessment method is conducted mainly in the form of questionnaires, such as the Cooper-Harper assessment questionnaire, the subjective load assessment, the Pearson fatigue scale, the Stanford sleep scale, the driver self-record table, the sleep habits questionnaire and so on. The objective assessment method is to record some changes in indicators like physiology, biochemistry, behavior of human body by means of instruments, equipment and other auxiliary tools. The comparison between subjective and objective methods is shown in the table1.

TABLE I. COMPARISON BETWEEN TWO METHODS

\begin{tabular}{|c|c|c|}
\hline & subjective & objective \\
\hline method & questionnaires & instrumentslequipment \\
\hline \multirow{4}{*}{ characteristics } & simple & complex \\
\cline { 2 - 3 } & no quantitative & quantitative \\
\cline { 2 - 3 } & unreliable & reliable \\
\cline { 2 - 3 } & non-real-time & real-time \\
\cline { 2 - 3 } & uncertainty & certainty \\
\hline
\end{tabular}

In this paper, the driver's eye characteristics are used as the basis for judging the fatigue state. First of all, the driver's operation is captured and inputted into the computer by the camera, at the same time, a simple image preprocessing is performed to improve the accuracy of detection. Then, the face area is located with AdaBoost face detection algorithm quickly. Next, the eye and the mouth state parameters can be extracted by constructing the driver's facial feature model with active shape model(ASM).

At last, with the establishment of the mapping from the eye characteristic state to the driver's fatigue state space using support vector method(SVM) algorithm, it can make a judgment of the driver's fatigue state. The detailed algorithm flow chart is shown in Figure 1 below.

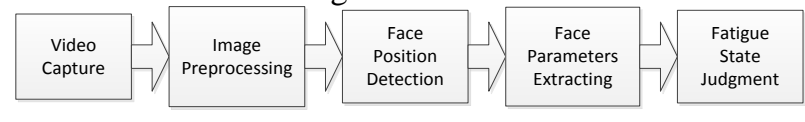

Figure 1. Flow chart of the detailed algorithm

\section{IMAGE PREPROCESSING AND FACE POSITIONING}

Due to the factors of acquisition equipment or human, there will always be more or less interference and noise in capturing the driver's face image, at the same time, the image of the color, background and lighting will also affect the accuracy of detection. To achieve a better detection results, it is necessary to perform an image preprocessing before extracting the parameters. The preprocessing operation is divided into three steps. The first step is to convert the color image captured by the camera into 8 bits gray images. Secondly, denoising the image with median filtering, which can remove the acnode noise but will not blur the boundary of the image. Finally, enhancing the contrast of the image with the histogram equalization.

Prepositioning the face area can reduce the complexity of extracting eye parameters and improve the efficiency of detection. The AdaBoost method is more suitable for the fast localization of human faces in terms of accuracy and running time. The AdaBoost algorithm is a method proposed by Viola and Jones in the paper named "real-time detection of face detection robustness"[8], which combines Haar-like eigen values and integral graphs to the detection of human faces. The basic principle is to construct simple Harr-like features into a weak classifier which can be used to form a strong classifier, and then combine several strong classifiers to construct a cascade classifier. If a rectangular box region in the detecting image can pass through the corresponding 
subclasses in the cascade classifier, it is assumed that the detecting image contains a human face and the position marked by the rectangle is where the face is. The figure2 shows the classifier with cascading.

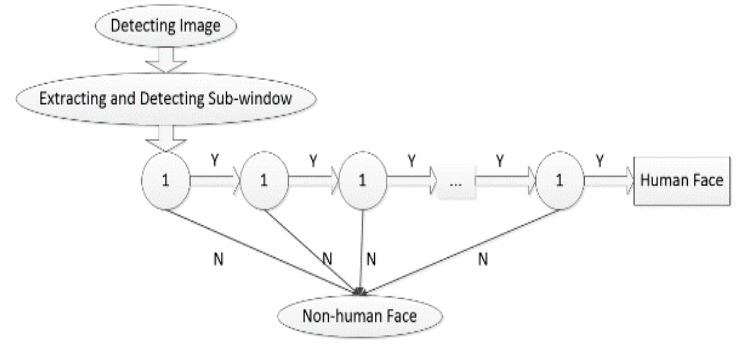

Figure 2. Classifier with cascading

The advantage of AdaBoost algorithm is that it can detect face stably, quickly and efficiently. The result of image preprocessing and face localization is shown in figure 3.

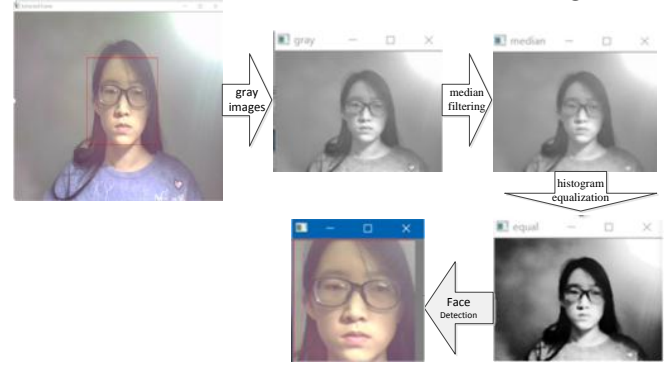

Figure 3. Result of image preprocessing andface localization

\section{MULTI-FEATURES POSITIONING}

In further recognition of the human eye's and mouth's position, the accuracy of the AdaBoost algorithm will be greatly reduced, which is not conducive to the judgment of the human eye state. In Point Distribution Models(PDM), the geometry of an object can be represented by a vector, which is composed of a number of key feature points in a given order, such as resistance, face, heart, and etc. As a PDM algorithm, ASM consists of two parts :model establishment and model matching, which is similar to most statistical learning methods.

\section{A. The establishment of ASM model}

Before using ASM, a large number of human faces need to be trained to obtain the initial shape model. The model training includes marking the feature points and extracting the shape models.

\section{1) Marking the feature points}

Selecting500 samples image of facial regions and marking feature points manually. These feature points are consistent with the standard 68 feature points provided by the ASM Library toolkit. Then, recording the coordinate of these 68 feature points and saving them in a text file.

\section{2) Extracting the shape model}

The shape vector of the 68 feature points marked in the sample image as:

$$
\alpha_{\mathrm{i}}=\left(\mathrm{x}_{0}^{\mathrm{i}}, \mathrm{y}_{0}^{\mathrm{i}}, \mathrm{x}_{1}^{\mathrm{i}}, \mathrm{y}_{1}^{\mathrm{i}}, \cdots, \mathrm{x}_{\mathrm{n}}^{\mathrm{i}}, \mathrm{y}_{\mathrm{n}}^{\mathrm{i}}\right), \mathrm{i}=1,2, \ldots, n,
$$

Where $\left(\mathrm{x}_{\mathrm{j},}^{\mathrm{i}} \mathrm{y}_{\mathrm{j}}^{\mathrm{i}}\right)$ represents the coordinates of the first $\mathrm{j}$ feature points on the first $\mathrm{i}$ training sample, $\mathrm{k}=0,1,2, \ldots, 67$; $\mathrm{n}=0,1,2, \ldots, 499$;

The feature point set is regarded as a two-dimensional vector:

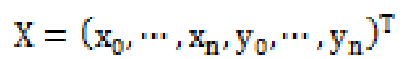

In order to compare the corresponding points from different images, the shape of the training set will be normalized to eliminate the non-shape interference caused by external factors, such as different angles of the face, the distance and the attitude change. Orthogonal Procrustes method can be used to align all the face shape vectors in the training set to the first shape vector with scaling, rotating and translating, but not changing the point distribution mode of the other shapes.

After the normalization of the data, principal component analysis (PCA)[9]algorithm is used to reduce dimension, which can reduce the computational complexity. Any set of feature points is regarded as a coordinate point in the principal component vector space. The coordinate origin is considered to be the mean of the set of points, so that any point is considered as the coordinate origin plus a vector:

$$
\mathrm{X} \approx \bar{X}+P
$$

\section{B. The matching of ASM models}

ASM matching is achieved by matching the shape parameters of the target image with an established model. First, each feature point in the trained shape model is initialized, giving an estimate to obtain the initial shape.

For any human face shape $\mathrm{X}$, its relation to $\overline{\mathbf{x}}$, 'P', 'b' can be expressed as:

$$
\begin{gathered}
\mathrm{X}=\mathrm{T}_{\mathrm{XY}_{\mathrm{Y}}, \theta}(\overline{\mathrm{x}}+\mathrm{Pb}) \\
\mathrm{T}_{\mathrm{XY}_{\mathrm{B}, \theta} \theta}\left(\begin{array}{l}
\mathrm{X} \\
\mathrm{y}
\end{array}\right)=\left(\begin{array}{l}
\mathrm{X}_{\mathrm{i}} \\
\mathrm{Y}_{\mathrm{i}}
\end{array}\right)+\left(\begin{array}{l}
\mathrm{s} \cos \theta-\mathrm{s} \sin \\
\mathrm{s} \sin \theta-\mathrm{s} \operatorname{co}
\end{array}\right.
\end{gathered}
$$

where $\mathrm{T}$ is the rotation scaling matrix, $(\mathrm{X}, \mathrm{Y})$ represents the horizontal, vertical translation change, $\mathrm{s}$ represents the scaling factor, and $\theta$ represents the rotation angle. At the time of initialization, the vector $b$ is zero, and the position of the best matching point is to be searched near the initial feature point by using the local gray model. The new shape model is renewed by updating the parameter $b$, and it can match with the corresponding feature point in the target image finally. The purpose of the match is to make the model $\mathrm{X}$ and the image feature point set $\mathrm{Y}$ nearest, which means to update the b until it converges.

The objective function is chosen to make the Euclidean distance of each point as small as possible. The Euclidean distance expression is:

$$
\mathrm{Z}=\left|\mathrm{Y}-\mathrm{T}_{\mathrm{XY}_{\mathrm{Y} \theta} \theta}(\overline{\mathrm{x}}+\mathrm{Pb})\right|^{2}
$$

ASM algorithm takes advantage of its point distribution model, calibrates facial information constantly in an iterative process, and finally locates the exact position of the human eye. Matching result is shown in Figure 4: 


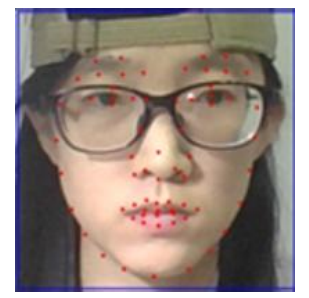

Figure 4. Result ofmatching

\section{EXTRACTION OF MULTI-FEATURESPARAMETERS}

Among all the dynamic changes in the human face, the change characteristics of the eyes and mouth are the most obvious. It is appropriate to use changes in the eye and mouth movements as a basis for detecting fatigue.

\section{A. EYE parameters}

In all the dynamic changes of facial features, eyes have the most significant features. The flow chart of eye feature parameters extraction.

\section{1) PERCLOSE parameter}

The U.S. highway traffic safety administration (NHTSA) shows that PERCLOS has the best correlation with drivers' fatigue levels by comparing nine indicators of fatigue[10]. PERCLOS refers to the percentage of eye closing time in a specific time, and NHTSA compares the evaluation criteria with PERCLOS, they believe that only if ones' degree of eye closure is more than $80 \%$ that can be regarded as the standard of eye closed (P80). Herein, the average human eye area of the driver in the normal state is calculated as the standard. Once the area of the driver's eye exceeds $80 \%$ of the standard, the driver is considered to be in an eye closed state. The results of eye opening and closing state recognition is shown in Figure 5.

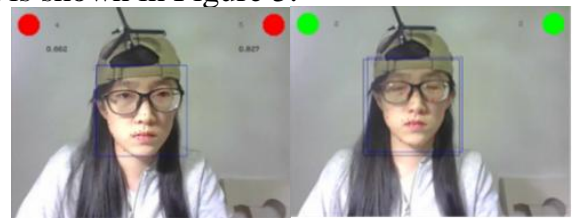

(a) eye opening

(b)eye closing

Figure 5. Results of eye opening and closing state recognition

In this paper, the number of frames in which the eyes are in closed state is denoted as $\mathrm{m} 1$, while total frame number of all frames is denoted as $\mathrm{n} 1$, the PERCLOS value can be expressed as:

$$
\text { PERCLOS }=\frac{\mathrm{m} 1}{\mathrm{n} 1}
$$

\section{2) BF parameter}

The BF parameter refers to the blinking frequency of the eye. According to standard P80, it can be considered as one blinking when the state of the eye is detected from opening to the closing, and then to opening. At the initial setting, the number of blinking is set to be zero. When the closing state is detected, the number of blinking plus one. Finally, the number of blinking in 60 seconds is recorded as $B_{n}$. Then the value of BF is:

$$
\mathrm{BF}=\frac{\mathrm{B}_{\mathrm{n}}}{60}
$$

\section{3) AECT parameter}

AECT represents the average time of eye closure, which is corresponding to the value of $\left(t_{2}-t_{2}\right)$ in the P80 principle. Denotingt $t_{i}$ as the time of the driver close his eye, the value of AECT can be obtained with the blink times $B_{\mathrm{n}}$, which is got in the process of BF parameter:

$$
\text { AECT }=\frac{\sum_{i=1}^{B n}\left(t_{1}+t_{2}+\cdots+t_{n}\right)}{B_{m}}, i=1,2, \ldots, B_{n}
$$

\section{B. MOUTH parameters}

Yawning is an important parameter of the human body when detecting fatigue, which is of great research value. Referring to the eye's PERCLOS principle, give a definition of PMRCLOS as a judgment standard for yawning in the mouth . the number of frames in which the mouthis in closed state is denoted as $\mathrm{m} 2$, while total frame number of all frames is denoted as n2, the PMRCLOS value can be expressed as:

$$
\text { PERCLOS }=\frac{\mathrm{m} 2}{\mathrm{n} 2}
$$

According to a large number of experimental data, when the ratio of mouth width to height exceeds 0.6 , it means that people are yawning.Theresults of mouth state recognition is shown in Figure 6.

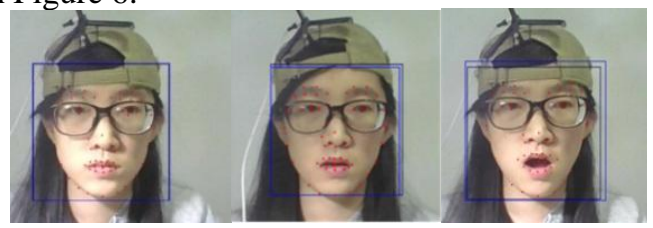

(a)mouth closing $\quad$ (b)talking

(c)yawning

Figure 6. Results of mouth state recognition

\section{FATIGUE JUDGMENT}

SVM is first proposed by Cortes and Vapnik in 1995, whichis based on VC dimension theory and structural risk minimization principle in statistical learning theory. In order to obtain the best generalization ability, SVM seeks the best compromise between model complexity and learning ability on a limited sample of information.

Compared with the traditional machine learning methods, SVM is used to solve the nonlinear and high dimensional mathematics problems with small sample, and has a strong ability to promote, which has been widely used in machine learning and other fields.

In this paper, a fatigue state detection model is built based on SVM. The construction process mainly includes three aspects:

\section{A. Model input}

Firstly, setting PERCLOS value, BF value, and AECT value as the inputted data of the support vector machine model, and get the characteristic parameter set $\mathrm{X}=$ 
[PERCLOS, BF, AECT,PMRCLOS]. Meanwhile, the optimal time window with characteristic parameters is set to bemorethan60 seconds. Next, extracting the eye movement characteristic parameters in a data segment of up to 60 seconds on both normal state and fatigue state. Finally, 50 samples of normal state and 50 groups of fatigue states are selected randomly to build a training sample database, and the remaining samples are used to build a test sample database.

\section{B. Model training}

Considering that the migration rule of the fatigue state is so complex rather than a simple linear problem. It is necessary to select a kernel function to classify the input of SVM model, which can map the data from the original space to the high-dimensional feature space. At present, the commonly used kernel functions are linear kernel function, polynomial kernel function, radial basis kernel function(RBF)and Sigmoid kernel function. Among them, RBF has some advantages in dealing with fatigue data detection[12]. The expression is as follows:

$$
\mathrm{k}\left(\mathrm{x}_{\mathrm{i}}, \mathrm{y}_{\mathrm{i}}\right)=\mathrm{e}^{-\mathrm{\gamma}\left|\mathrm{x}_{\mathrm{i}}-\mathrm{x}_{\mathrm{i}}\right|^{*}}
$$

There are two pending variables in the process of model building: the penalty coefficient $C$ and the kernel variable $\gamma$. The penalty coefficient $\mathrm{C}$ controls the recognition accuracy and generalization ability of the fatigue detection model. The kernel variable $\gamma$ determines whether it is linearly separable when the nonlinear problem in the original space is transformed into a high dimensional space.

\section{Model test}

The SVM fatigue state detection model is implemented with open source software package LIBSVM. To validate the effective of the proposed algorithm, it utilizes the remaining 50 sets of normal driving samples and 50 sets of fatigue driving samples to form a test set. The results of the verification are shown in table II:

TABLE II. RESULTS OF THE VERIFICATION

\begin{tabular}{|c|c|c|}
\hline \multirow{2}{*}{ Model Output } & \multicolumn{2}{|c|}{ Actual State } \\
\cline { 2 - 3 } & Normal Status & Fatigue state \\
\hline Normal Status & 42 & 6 \\
\hline Fatigue State & 8 & 44 \\
\hline
\end{tabular}

\section{CONCLUSION}

This paper focuses on the relationship between face characteristics and fatigue state of drivers. It integrates various of parameters, including eyes' and mouth', which improves the reliability of system. With building a fatigue monitoring model with multiple types of characteristic parameters, the system can finally determine whether the driver is in fatigue.

Experimental data shows that the precision of the system reaches to $86 \%$, which indicates the high accuracy of the test.
The research results of the thesis can provide support for the fatigue monitoring technology to the operator in a certain extent.

\section{ACKNOWLEDGMENT}

This paper is supported by the local special program of the Shaanxi Provincial Department of Education (No. 16JF012), the National Natural Science Foundation of China (No. 61572392).

\section{REFERENCES}

[1] Wendong Cheng, Rui Fu, Wei Yuan, Yingshi Guo, The driver fatigue monitoring technology research and Application,vol.01.China Safety Science Journal, 2013, pp.155-160.

[2] Yangyang Zou, and Guoxun King,Experimental Study on Comprehensive Index of Driving Fatigue Based on Physiological Signals, vol.03. Journal of safety and environment, 2015, pp.57-61.

[3] Hongyu Hu, "The steering driving fatigue detection analysis method of wheel angle speed time series," 2015 Automotive Engineering Society Annual Conference, vol. 2, pp.3-13, 2015.

[4] Wei Zhang, Research on the Key Problem of Driver's Fatigue State Recognition Based on Machine Vision, Tsinghua University, 2011.

[5] Cevikalp H,Triggs B, and France V, "Face and landmark detection by using cascade of classifiers,"2013 10th IEEE International Conference and Workshops on, pp.1-7, 2013.

[6] Joseph S,Sowmiya R, and Thomas R A, "Face detection through nerual network," Current Trends in Engineering and Technology (ICCTET), pp.163-166, 2014

[7] Wei Gu,Wenjie Liu, and Haozhong Zhu,Research on face recognition algorithm based on Bayesian multi classifier fusion, 13th ed., vol.12. Software Guide,2014, pp.65-67.

[8] VIOLA P, and JONES M J, Robust real-time face detection, 57th ed., vol.2. International Journal of Computer Vision, 2004, pp.137-154

[9] V. Kecman, Learning and Soft Computing, Support Vector machines, Neural Networks and Fuzzy Logic Models, The MIT Press, Cambridge, MA, 2001.

[10] DINGES D F, and GRACE R, Perclos: A Vaid Psychomotor Vigilance.US Department of Transportation. Federal HighWay Administration, 1988.

[11] JIN L S, NIU Q N, and HOU H J, Driver Cognitive Dis-traction Detection Using Driving Performance Measure, vol.2012.Discrete Dynamics in Nature and Society,2012, pp.1-12.

[12] Liu Liu, white Zhonghao, Jiao Kennedy, and Cao song, Multi Feature Fusion ASM Driver Fatigue Detection Methods,VOL.12. Journal of Electronic Measurement and Instrument, 2016, pp.1877-1883.

[13] Wang Mei,Guo Lin, and Chen Wen-Yuan. Blink Detection Using Adaboost and Contour Circle for Fatigue Recognition, vol.58. Computers and Electrical Engineering, v 58, February 2017, pp.502512.

[14] Zheng Chang, Xiaojuan Ban, and Yu Wang, Fatigue Driving Detection based on Haar Feature and Extreme Learning Machine, 4th ed., vol.23. Journal of China Universities of Posts and Telecommunications, August 2016, pp.91-100.

[15] Likun Zhao, Xinhua Jiang, and Haiyan Yang, Driver Fatigue Detection based on MLBPH-FF and SVM, vol.09. Computer and Modernization, 2013, pp.82-85.

[16] Zhiqiang Zhou, Qingling Niu, Lisheng Jin, Wenchao Liu, and Pengcheng Yu, On Detection Method of Fatigue Driving Characteristics of Eye Movement,vol.03. Journal of Harbin Engineering University, 2015, pp.394-398. 